\title{
Hermite-Hadamard type inequalities for multiplicatively geometrically $P$-functions
}

\author{
Date of Receiving : $\quad 11.12 .2018$ \\ Date of Revision : $\quad 25.12 .2018$ \\ Date of Acceptance : 25.12 .2018
}

MAHIR KADAKAL ${ }^{\dagger}$, HALIL KARACA AND IMDAT IŞCAN

\begin{abstract}
In this paper, we introduce a new class of extended multiplicatively geometrically $P$-function. Some new Hermite-Hadamard type inequalities are derived. Results represent significant refinement and improvement of the previous results.
\end{abstract}

\section{Preliminaries and Fundamentals}

Definition 1.1. A function $f: I \subseteq \mathbb{R} \rightarrow \mathbb{R}$ is said to be convex if the inequality

$$
f(t x+(1-t) y) \leq t f(x)+(1-t) f(y)
$$

is valid for all $x, y \in I$ and $t \in[0,1]$. If this inequality reverses, then the function $f$ is said to be concave on interval $I \neq \emptyset$.

This definition is well known in the literature. Convexity theory has appeared as a powerful technique to study a wide class of unrelated problems in pure and applied sciences.

One of the most important integral inequalities for convex functions is the HermiteHadamard inequality. The classical Hermite-Hadamard inequality provides estimates of the mean value of a continuous convex function $f:[a, b] \rightarrow \mathbb{R}$. The following double inequality is well known as the Hadamard inequality in the literature.

Definition 1.2. $f:[a, b] \rightarrow \mathbb{R}$ be a convex function, then the inequality

$$
f\left(\frac{a+b}{2}\right) \leq \frac{1}{b-a} \int_{a}^{b} f(x) d x \leq \frac{f(a)+f(b)}{2}
$$

is known as the Hermite-Hadamard inequality.

2010 Mathematics Subject Classification. 26A51, 26D10, 26D15.

Key words and phrases. Convex function, Multiplicatively $P$-function, Multiplicatively Geometrically $P$-function, Hölder Integral inequality and Power-Mean Integral inequality, Hermite-Hadamard type inequality.

Communicated by: Nikhil Khanna

${ }^{\dagger}$ Corresponding author. 